\title{
Miniaturized High-Order-Mode Dipole Antennas Based on Spoof Surface Plasmon Polaritons
}

\author{
Yijuan Yang, Zheng Li, Member, IEEE, Shanzhe Wang, Xuyang Chen, Junhong Wang, Senior Member, IEEE, and Y. Jay Guo,
} Fellow, IEEE

\begin{abstract}
A miniaturization method for antennas is developed based on spoof surface plasmon polaritons (SSPPs). With a large phase constant on the SSPPs transmission line, the guide wavelength can be dramatically reduced, with great potential on the miniaturization application of antennas. By introducing a miniaturization factor $M$, the relationship between $M$ and the phase constant on the SSPPs dipole is studied, providing a guidance to the design of miniaturized SSPPs dipoles. Then SSPPs dipoles operating at $2.4 \mathrm{GHz}$ are designed, including both the oddand even-resonance dipoles. Simple feeding structures are developed for the odd- and even-resonance dipoles, respectively, and particularly for the even-resonance mode to realize a transition from $50 \Omega$ to a very large input impedance of the dipole. The miniaturized SSPPs dipoles operating at the $1^{\text {st }}, 2^{\text {nd }}, 3^{\text {rd }}$, and $4^{\text {th }}$ modes are fabricated. The measured reflection coefficients and radiation patterns show good agreements with the simulated results. It can be concluded that the dipole lengths for these modes are reduced by $8 \%, 11 \%, 10 \%, 13 \%$, respectively, compared with the conventional printed dipoles on the same substrate.
\end{abstract}

Index Terms - Miniaturization, spoof surface plasmon polaritons, dipole, resonance modes.

\section{INTRODUCTION}

$\mathrm{S}_{\mathrm{p}}^{\mathrm{P}}$ POOF surface plasmon polaritons (SSPPs) has attracted much interest in the electromagnetics community since the idea was inspired from the surface plasmon polaritons (SPPs) in optical band [1] [2]. It is a type of slow-wave transmission line (TL) with tightly confined waves on it, and has been widely investigated in microwave and millimeter-wave frequency bands. In [3]-[6] the SSPPs is employed in the design of a single-conductor TL without metal ground plane. In [7]-[9] leaky-wave antennas are developed based on SSPPs TL with periodic perturbations, and hence frequency-beam scanning can be realized. SSPPs structures are also employed in the designs of end-fire antennas and dielectric resonator antennas as the feeding line [10] [11], and also in the antenna array as the feeding network [12]. Besides, SSPPs have also been studied for microwave components, such as power dividers [13], filters

Manuscript received on July 19, 2018. This work was supported by the Fundamental Research Funds for the Central Universities under grant 2018JBZ102 and 2017YJS015, the National Natural Science Foundation of China under Grant 61771038 and 61331002, and the Australia Research Council under Grant DP160102219. (Corresponding Author: Zheng Li)

Y. J. Yang, Z. Li, S. Z. Wang, and J. H. Wang are with the Key Laboratory of All Optical Network and Advanced Telecommunication Network of Ministry of Education, and the Institute of Lightwave Technology, Beijing Jiaotong University, Beijing 100044, China (e-mail: lizheng@bjtu.edu.cn).

$\mathrm{X}$. Y. Chen is with the Beijing NAURA Microelectronics Equipment Co.,Ltd.

Y. J. Guo is with the Global Big Data Technologies Centre, University of Technology Sydney, Ultimo, NSW 2007, Australia.
[14], [15], and couplers [16]. Although the SSPPs have been widely studied in many areas, most of the reported research is based on the travelling-wave transmission mechanism. In other words, the application of SSPPs in the standing-wave resonance structures has not been investigated to the authors' best knowledge. In addition, the mode propagating on the SSPPs is slow wave, with large phase constant and very small wavelength, thus providing great potential in miniaturization applications. In this paper, therefore, we explore a new application of SSPPs for the miniaturization of resonant antennas.

There are many types of standing-wave based antennas, in which the dipole is the most common one [17]-[21], and has been extended to many types of antennas such as Yagi-Uda [22], [23], and folded dipoles [24]. In [18] a compressed dipole is proposed based on dielectric slab with high permittivity, and higher order modes are studied for improved boresight directivity. However, it is not convenient to use substrates with different permittivity in a practical integrated circuit board. In this paper, therefore, we develop a new way to realize the miniaturization of dipole antennas using an SSPPs structure.

The modes of the dipole can be categorized into two types: odd-resonance modes and even-resonance modes. Among the odd-resonance modes, only the half-wave dipole has been widely used due to its simple structure and easy feeding. For even-resonance modes, however, one faces the main problem of high input impedance (up to hundreds of Ohms), making the feeding difficult. Some approaches have been reported for solving the feeding problem. In [19] offset feeding is proposed for full-wave dipole, and the feeding point is away from the center of the dipole, where the current reaches the peak value. However, the offset feeding has a bad effect that yields splitted beam. In [20] dual-/triple-feeding is used to improve the radiation performance of the full-wave dipole, but with higher complexity and more difficulty for integration. Recently, a hybrid feeding method is proposed in [21], consisting of a triple-tuned circuit and impedance transformer to realize wideband matching for full-wave dipoles. Most of the research is focused on full-wave dipoles, and dipoles operating at higher order modes have been rarely studied due to the large length and difficulties in matching.

In this paper, taking dipole antenna as an example, the miniaturization of antennas based on SSPPs is studied, which is a new perspective for the application of SSPPs. A large phase constant is generated on the SSPPs to achieve miniaturization ability. High-order-mode SSPPs dipoles are presented, and simple matching method is developed for the matching of both of the odd- and even-resonance modes. 


\section{PRinCiPle of Miniaturization BASEd ON SSPPS}

The geometry of the miniaturized SSPPs dipole is depicted in Fig. 1. It is printed on a TLX-8 substrate with dielectric constant $\varepsilon_{r}=2.55$, loss tangent $\tan \delta=0.0019$, and thickness of $1.575 \mathrm{~mm}$. The operating frequency is chosen to be $2.4 \mathrm{GHz}$. For the unit cell of SSPPs structure, $p$ represents the period length of the serrated stubs, $b$ is the stub width, and $h$ is the stub length. According to [3], [5], [6], the phase constant $\beta$ on the SSPPs is mainly determined by $h$, while $p$ and $b$ have much smaller effects on $\beta$. In this paper full-wave simulations are executed to examine their relationships using the commercial electromagnetic simulation software CST Microwave Studio.
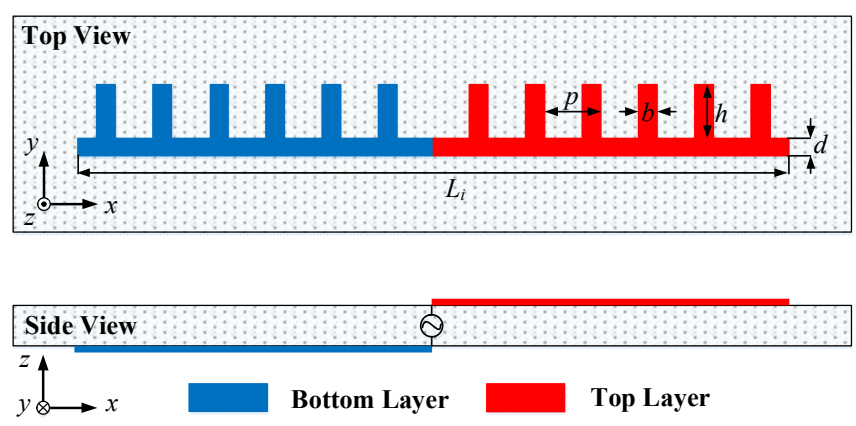

Fig.1. Configuration of the miniaturized SSPPs dipole.

In order to find the relationship between $\beta$ and $h$, a travelling-wave SSPPs TL is employed with varied $h$ but fixed $p(3 \mathrm{~mm})$ and $b(1 \mathrm{~mm})$. Then the variation of $\beta$ with $h$ at 2.4 $\mathrm{GHz}$ is obtained, as shown in Fig. 2. It can be seen that the value of $\beta$ increases from $56.7 \mathrm{rad} / \mathrm{m}$ to $79.1 \mathrm{rad} / \mathrm{m}$ when $h$ changes from $0 \mathrm{~mm}$ to $11 \mathrm{~mm}$, showing an obvious upward trend. Thus a smaller guide wavelength along the SSPPs TL can be produced. It has great potential on the reduction of the resonance length for the dipole antenna, and hence yields miniaturization. However, as shown in Fig.2 the increase of $\beta$ slows down gradually, especially when $h$ is larger than $8 \mathrm{~mm}$, because there is a physical limit for the increase of $\beta$.

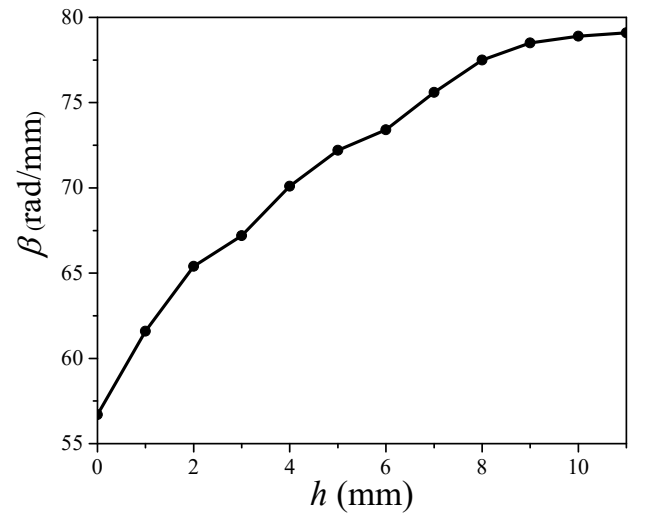

Fig. 2. Relationship between $\beta$ and $h$ at $2.4 \mathrm{GHz}$.

A minimization factor $M$, similar to that in [18], is introduced for the investigation on the miniaturization ability of SSPPs, as expressed in Eq.(1):

$$
M=L_{i} / L_{0 i} \approx \beta_{0} / \beta
$$

where $L_{i}$ and $L_{0 i}$ represent the resonance length of the $i$ th order mode of SSPPs dipole and that of conventional printed dipole on the same substrate, respectively. $\beta_{0}$ is the phase constant on the arm of the conventional printed dipole without serrated stubs $(h=0 \mathrm{~mm})$. In fact, $M$ is not exactly equal to the ratio of $\beta_{0}$ to $\beta$ due to the following reasons: First, the two arms of the dipole are on different layers with a gap between them for excitation, as depicted in Fig.1; Second, due to the serrated stubs, the arms of SSPPs dipole have larger arm width and stronger distortion on the current distribution, yielding more obvious distinction from that of the conventional dipole. Based on the above analysis, the relationship between $M$ and $\beta$ of the miniaturized SSPPs dipole operating at different resonance modes are studied, as demonstrated in Fig. 3. From Fig.3, we can draw the following conclusions: (i) The minimization factor $M$ decreases when $\beta$ increases, demonstrating that the resonance length of SSPPs dipole becomes shorter owing to the reduced guide wavelength along the SSPPs structure; (ii) There are differences in the values of $M$ for each mode even though they have the same $\beta$. That is why Eq.(1) only shows an approximate relationship; (iii) The value of $M$ for the even-resonance mode is smaller than that of the adjacent lower order odd-resonance mode. For instance, the $2^{\text {nd }}$ order mode has smaller $M$ than the $1^{\text {st }}$ mode, demonstrating stronger miniaturization ability. Therefore, using Fig.3 we can achieve desired $M$ by choosing proper $\beta$, and furthermore the serrated stub length $h$ can be determined from Fig.2. For example, for the $1^{\text {st }}$ mode, if we need a length reduction of $21 \%(M=0.79)$ compared with that of the conventional printed dipole, $\beta$ should be $75.6 \mathrm{rad} / \mathrm{m}$ according to Fig. 3 , and the corresponding stub length $h$ should be $7 \mathrm{~mm}$ according to Fig.2. However, we found that if $h$ is larger than $8 \mathrm{~mm}$, it will have negative effect on the radiation performance, yielding a distortion on the radiation pattern. In this paper, in order to show the miniaturization method whilst maintaining good radiation performance of the SSPPs dipole antenna, we choose the value of $h$ to be $2 \mathrm{~mm}$ in the following designs, so the corresponding $\beta$ is $65.4 \mathrm{rad} / \mathrm{m}$. Then the values of $M$ for all the eight modes are summarized in Table I.

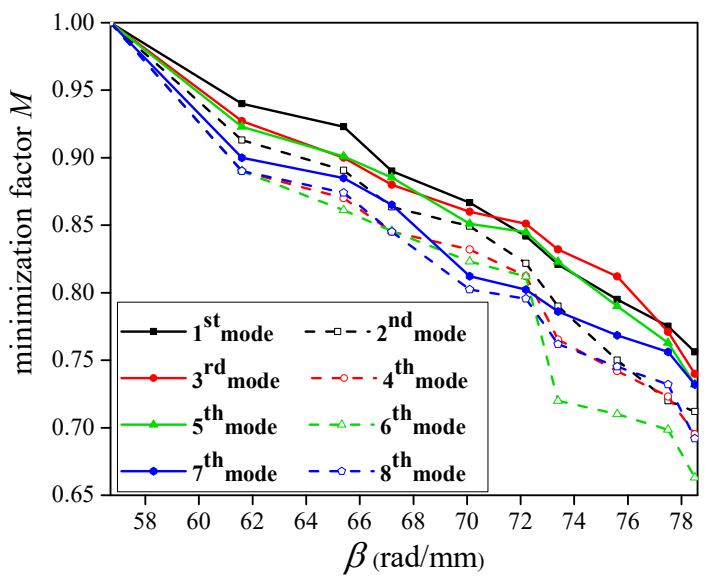

Fig. 3. Relationships between $M$ and $\beta$ for the SSPPs dipoles at different resonance modes at $2.4 \mathrm{GHz}$.

Table I. The minimization factor $M$ of the SSPPs dipoles with different modes.

\begin{tabular}{|c|c|c|c|c|c|c|c|c|}
\hline Modes & $1^{\text {st }}$ & $2^{\text {nd }}$ & $3^{\text {rd }}$ & $4^{\text {th }}$ & $5^{\text {th }}$ & $6^{\text {th }}$ & $7^{\text {th }}$ & $8^{\text {th }}$ \\
\hline$M$ & $92 \%$ & $89 \%$ & $90 \%$ & $87 \%$ & $90 \%$ & $86 \%$ & $89 \%$ & $87 \%$ \\
\hline
\end{tabular}




\section{Matching Method AND Results}

The input impedances and feeding method of the miniaturized SSPPs dipoles are studied in this section. The structural parameters in Fig.1 remain unchanged in this section ( $p=3 \mathrm{~mm}, b=1 \mathrm{~mm}, h=2 \mathrm{~mm}$ ), while the arm lengths $L_{i}$ are different for different resonance modes.

\section{A. Odd-Resonance-Mode SSPPs Dipoles}

The input impedances of the miniaturized SSPPs dipoles for the odd-resonance modes are shown in Fig.4. It can be seen that the real parts are around $50 \Omega$, and the imaginary parts are close to $0 \Omega$ at $2.4 \mathrm{GHz}$ for all the resonance modes. However, the higher-order modes $\left(5^{\text {th }}, 7^{\text {th }}\right)$ have more obvious fluctuation in the observation band. It can be inferred that the same feeding structure can be used for all the odd-resonance modes at 2.4 $\mathrm{GHz}$ owing to similar input impedances. Then a microstrip line with tapered ground is designed to provide balanced feeding for the two arms in Fig.5. An isosceles right angle triangle is cut at the end of the feeding line for better matching with the SSPPs arms. To verify the proposed designs, the SSPPs dipoles of the $1^{\text {st }}$ and $3^{\text {rd }}$ modes are fabricated, as shown in Fig.5.

Fig. 6 depicts the reflection coefficients, demonstrating that all the dipoles with four different resonance modes have the same resonance frequency $2.4 \mathrm{GHz}$. The reflection coefficients are less than $-20 \mathrm{~dB}$ for all the modes at $2.4 \mathrm{GHz}$. The measured reflection coefficients for the $1^{\text {st }}$ and $3^{\text {rd }}$ modes match well with the simulated results, which verifies the proposed design.

The simulated and measured radiation patterns of gain at 2.4 $\mathrm{GHz}$ are depicted in Fig. 7. The values of simulated gain for the $1^{\text {st }}$ and $3^{\text {rd }}$ modes are $2.0 \mathrm{dBi}$ and $3.9 \mathrm{dBi}$, respectively, while the measured results are $1.9 \mathrm{dBi}$ and $3.7 \mathrm{dBi}$, respectively. The cross polarization level is less than $-14 \mathrm{dBi}$ for both modes. Good agreement is observed between the simulated and measured results.

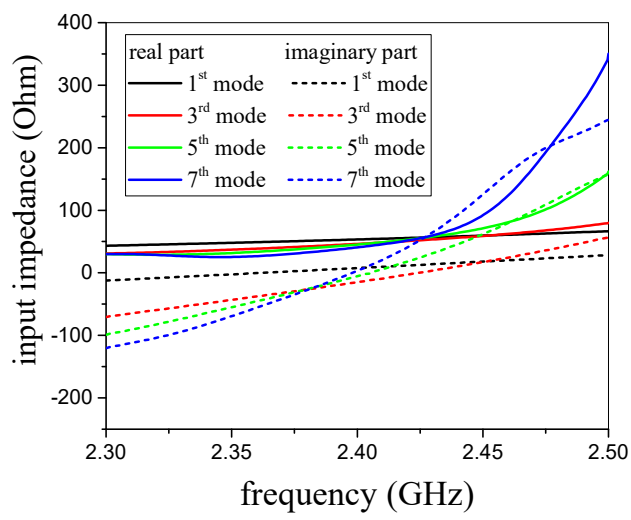

Fig. 4. Input impedances of the miniaturized SSPPs dipoles (without feeding line) operating at odd-resonance modes.

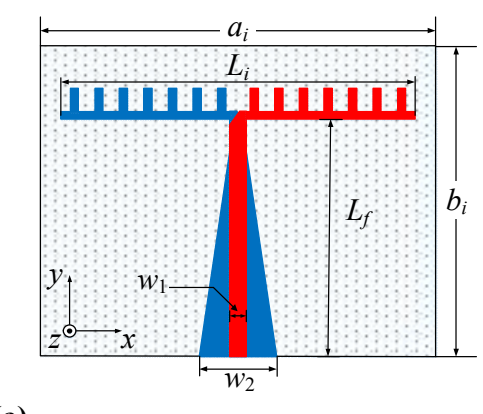

(a)
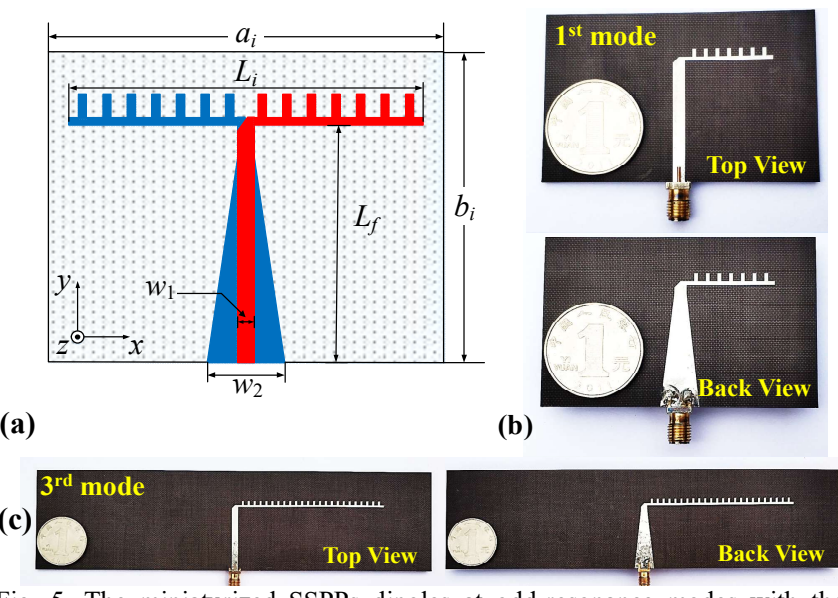

Fig. 5. The miniaturized SSPPs dipoles at odd-resonance modes with the feeding structure. (a) Configuration of the SSPPs dipole. (b) Prototypes of the fabricated dipoles. Structure parameters $(\mathrm{mm}): L_{f}=33.2, w_{1}=3.2, w_{2}=10$. The arm length $L_{i}$ for each mode $(\mathrm{mm}): L_{1}=45.2, L_{3}=147.6, L_{5}=240.7, L_{7}=332.3$. The length $a_{i}$ for all the odd-resonance mode dipoles (mm): $a_{1}=70, a_{3}=200, a_{5}$ $=280, a_{7}=400$. The width $b_{i}$ for all the odd-resonance mode dipoles $(\mathrm{mm}): b_{1}=$ $45, b_{3}=45, b_{5}=45, b_{7}=45$.

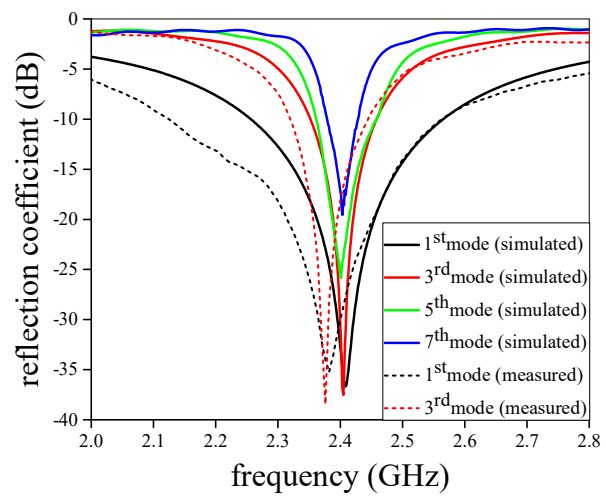

Fig. 6. Reflection coefficients of the odd-resonance-mode SSPPs dipoles.

\section{B. Even-Resonance-Mode SSPPs Dipoles}

Fig. 8 shows the input impedances of the miniaturized SSPPs dipole for the even-resonance modes. It can be observed that the SSPPs dipoles operating at different even-resonance modes have the similar real parts of the input impedance around $590 \Omega$, much higher than that of the conventional even-resonance-mode dipoles, while the imaginary parts are around $0 \Omega$. This makes it more difficult to design a feeding structure for the SSPPs dipoles, and the conventional microstrip TL is almost useless for this case. Therefore, in this paper a two-step transition is developed, consisting of a tapered microstrip TL and a tapered SSPPs TL, as shown in Fig.9. In the section of microstrip TL, the line width is gradually reduced to generate high characteristic impedance. In the section of
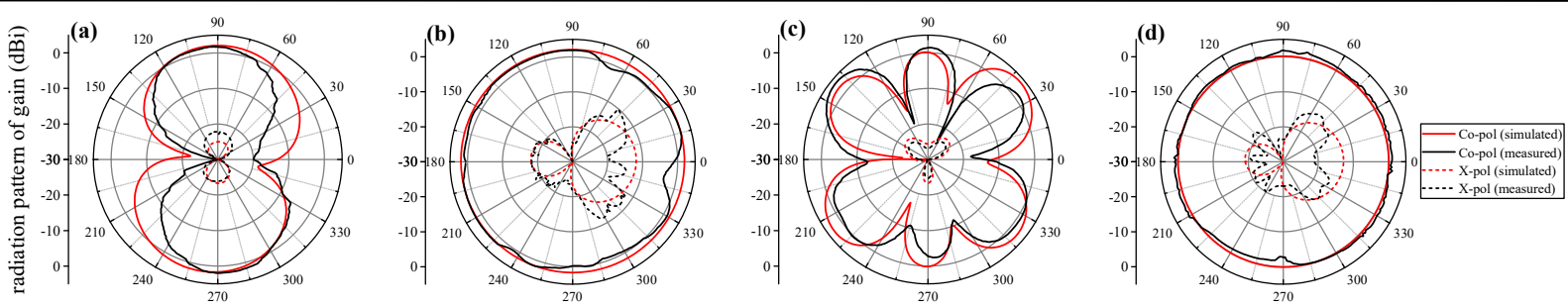

Fig. 7. Simulated and measured radiation patterns of gain at $2.4 \mathrm{GHz}$. (a) E- plane of the $1^{\text {st }}$-resonance-mode dipole. (b) H-plane of the $1^{\text {st }}$-resonance-mode dipole. (c) E-plane of the $3^{\text {rd }}$-resonance-mode dipole. (d) H-plane of the $3^{\text {rd }}$-resonance-mode dipole. 
tapered SSPPs TL, the serrated stub length $h_{f}$ is gradually increased from 0.5 to $2.4 \mathrm{~mm}$, while $b_{f}, p_{f}$ and $w_{f}$ remain unchanged $\left(b_{f}=1 \mathrm{~mm}, p_{f}=3 \mathrm{~mm}, w_{f}=0.2 \mathrm{~mm}\right)$. Thus this feeding structure can realize high characteristic impedance to match with the input impedance of the even-resonance modes. To validate the proposed designs, the SSPPs dipoles of the $2^{\text {nd }}$ and $4^{\text {th }}$ modes are fabricated, as shown in Fig. 9.

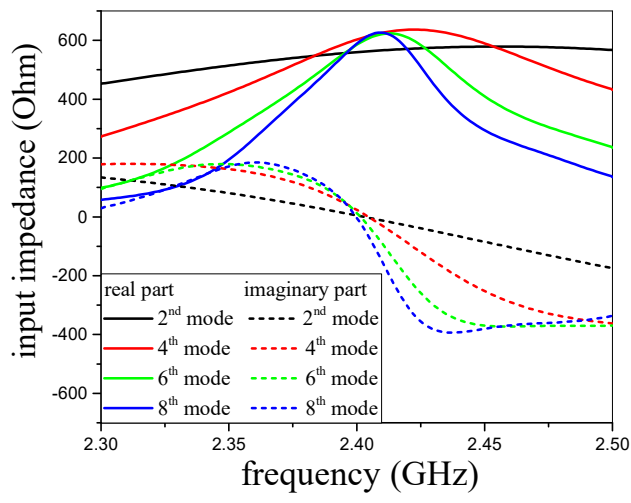

Fig. 8. Input impedances of the miniaturized SSPPs dipoles (without feeding line) operating at even-resonance modes.
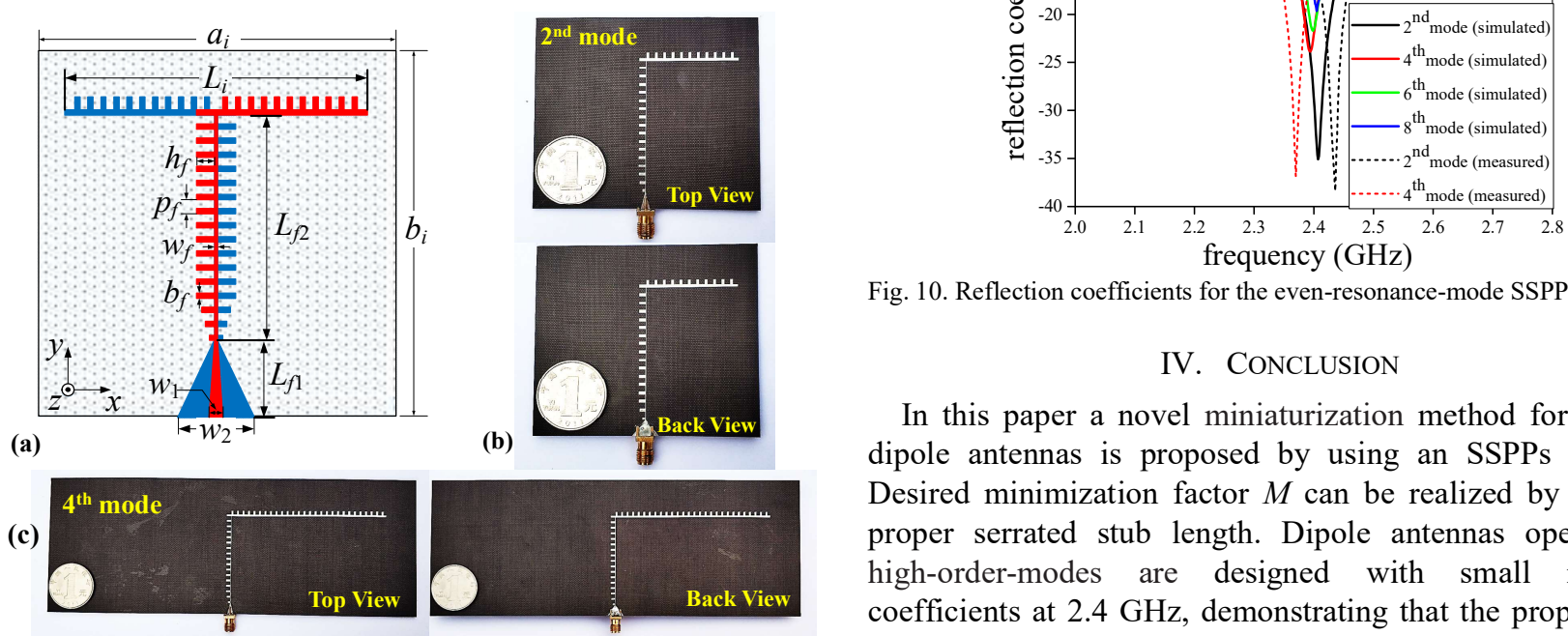

Fig. 10. Reflection coefficients for the even-resonance-mode SSPPs dipoles.

\section{CONCLUSION}

In this paper a novel miniaturization method for resonant dipole antennas is proposed by using an SSPPs structure. Desired minimization factor $M$ can be realized by choosing proper serrated stub length. Dipole antennas operating at high-order-modes are designed with small reflection coefficients at $2.4 \mathrm{GHz}$, demonstrating that the proposed two matching structures are versatile for the odd- and even-resonance modes, respectively. When the stub length is 2 $\mathrm{mm}$, the dipole lengths at these modes are reduced by $8 \%, 11 \%$, $10 \%, 13 \%$, respectively, compared with the conventional printed dipoles on the same substrate. The antenna can be further minimized if smaller $M$ is chosen. The antennas are fabricated, and the experimental results have verified the performance of the simulated designs.

The reflection coefficients are depicted in Fig. 10. It can be observed that the reflection coefficients are less than $-20 \mathrm{~dB}$ for all the four even-resonance-mode dipoles $\left(2^{\text {nd }}, 4^{\text {th }}, 6^{\text {th }}, 8^{\text {th }}\right)$ at the
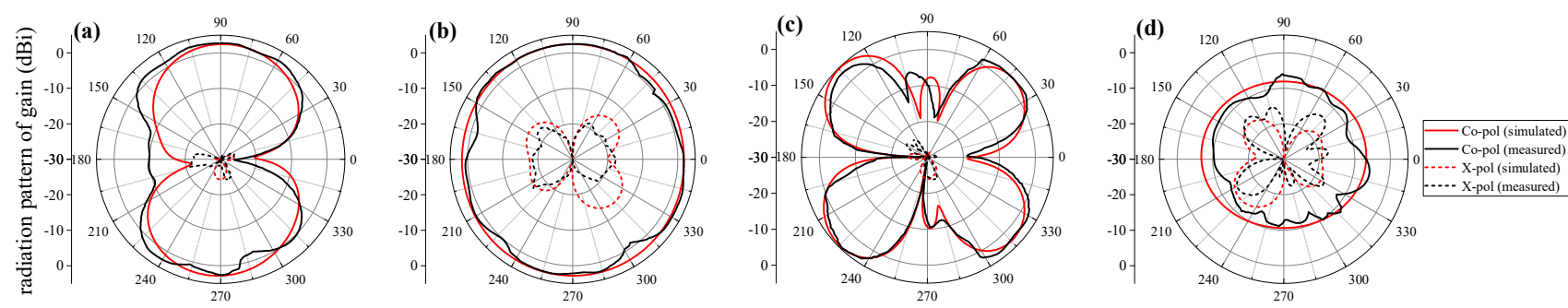

Fig. 11. Simulated and measured radiation patterns of gain at $2.4 \mathrm{GHz}$. (a) E- plane of the $2^{\text {nd }}$-resonance-mode dipole. (b) $\mathrm{H}$-plane of the $2^{\text {nd }}$-resonance-mode dipole. (c) E-plane of the $4^{\text {th }}$-resonance-mode dipole. (d) H-plane of the $4^{\text {th }}$-resonance-mode dipole. 


\section{REFERENCES}

[1] W. L. Barnes, A. Dereux, and T. W. Ebbesen, "Surface plasmon subwavelength optics," Nature, vol. 424, pp. 824-830, 2003.

[2] J. Pendry, L. Martin-Moreno, and F. Garcia-Vidal, "Mimicking surface plasmons with structured surfaces," Science, vol. 305, no. 5685, pp. 847-848, Aug. 2004.

[3] H. F. Ma, X. Shen, Q. Cheng, W. X. Jiang, and T. J. Cui, "Broadband and high-efficiency conversion from guided waves to spoof surface plasmon polaritons," Laser Photon. Rev., vol. 8, no. 1, pp. 146-151, 2014.

[4] X. R. Zhang, H. C. Zhang, W. X. Tang, J. F. Liu, Z. Q. Fang, J. W. Wu, T. J. Cui, "Loss Analysis and Engineering of Spoof Surface Plasmons Based on Circuit Topology," IEEE Antennas Wireless Propag. Lett., vol. 16, pp. 3204 - 3207, Nov. 2017.

[5] A. Kianinejad, Z. N. Chen, and C. W. Qiu, "Design and modeling of spoof surface plasmon modes-based microwave slow-wave transmission line," IEEE Trans. Microw. Theory Techn., vol. 63, no. 6, pp. 1817-1825, Jun. 2015.

[6] A. Kianinejad, Z. N. Chen, L. Zhang, W. Liu, and C. W. Qiu, "Low-loss spoof surface plasmon slow-wave transmission lines with compact transition and high isolation ,"IEEE Trans. Microw. Theory Techn., vol. 64, no. 10, pp. 3078-3086, Oct. 2016

[7] Q. L. Zhang, Q. F. Zhang, and Y. F. Chen, "Spoof surface plasmon polariton leaky-wave antennas using periodically loaded patches above PEC and AMC ground planes," IEEE Antennas Wireless Propag. Lett., vol. 16, pp. 3014-3017, Nov. 2017.

[8] J. Y. Yin, J. Ren, Q. Zhang, H. C. Zhang, Y. Q. Liu, Y. B. Li and T. J. Cui, "Frequency-controlled broad-angle beam scanning of patch array fed by spoof surface plasmon polaritons," IEEE Trans. Antennas Propag., vol. 64, no. 12 , pp. 5181-5189, Dec. 2016.

[9] G. S. Kong, H. F. Ma, B. G. Cai, and T. J. Cui, "Continuous leaky-wave scanning using periodically modulated spoof plasmonic waveguide," Sci. Rep., vol. 6, $29600,2016$.

[10] J. Y. Yin, D. Bao, J. Ren, H. C. Zhang, B. C. Pan, Y. F. Fan and T. J. Cui, "Endfire radiations of spoof surface plasmon polaritons," IEEE Trans. Microw. Theory Techn., vol. 16, pp. 597-600, Mar. 2017.

[11] A. Kianinejad, Z. N. Chen, L. Zhang, W. Liu, and C. W. Qiu, "Spoof plasmon-based slow-wave excitation of dielectric resonator antennas," IEEE Trans. Antennas Propag., vol. 64, no. 6, pp. 2094-2099, Jun. 2016.

[12] C. Zheng, H. Yi, and S. H. Qu, "High-gain and wideband antenna arrays," IEEE Antennas Progag. Mag., vol. 58, pp. 22-34, Aug. 2016.

[13] Y. L. Wu, M. X. Li, G. Y. Yan, L. Deng, Y. A. Liu, and Z. Ghassemlooy, "Single-conductor co-planar quasi-symmetry unequal power divider based on spoof surface plasmon polaritons of bow-tie cells," AIP Advances, vol.6, 105110, 2016.

[14] $\mathrm{Hu}, \mathrm{M}$. Z. et al., "Ultra-wideband filtering of spoof surface plasmon polaritons using deep subwavelength planar structures," Sci. Rep. 6, 37605; doi: 10.1038/srep37605 (2016).

[15] B. C. Pan and T. J. Cui, "Broadband Decoupling Network for Dual-Band Microstrip Patch Antennas," IEEE Trans. Antennas Propag., vol. 65, no. 10, pp. 5595-5598, Oct. 2017.

[16] X. Y. Liu, Y. J. Feng, K. Chen, B. Zhu, J. M. Zhao, and T. Jiang, "Planar surface plasmonic waveguide devices based on symmetric corrugated thin film structures," Optics Express, vol. 22, no. 17, pp. 20107-20116, Aug. 2014.

[17] C. A. Balanis, "Linear Wire Antennas," in Antenna Theory: Analysis and Design, 3rd ed., Wiley, Hoboken, NJ, 2005.

[18] Y. Luo, and Z. N. Chen, "Compressed dipoles resonating at higher order modes with enhanced directivity," IEEE Trans. Antennas Propag., vol. 65, pp. 5697-5701, Nov. 2017.

[19] S. P. Kosta, M. D. Singh, and R. P. Agarwal, "Theoretical investigations on the centre-fed full-wave dipole antenna with feed-points displaced along the axis of the dipole," IEE-IERE Proc. India, vol. 8, pp. 56-59, Apr. 1970.

[20] Y. Khraisat, K. Hmood, and A. Anwar, "Analysis of the radiation resistance and gain of full-wave dipole antenna for different feeding design," J. Electromagn. Anal. Appl., vol. 4, pp. 235-242, 2012.

[21] C. Ding, B. Jones, Y. J. Guo, and P. Y. Qin, "Wideband matching of full-wavelength dipole with reflector for base station," IEEE Trans. Antennas Propag., vol. 65, no. 10, pp. 5571-5576, Oct. 2017.

[22] D. M. Pozar, "Beam transmission of ultra short waves: An introduction to the classic paper by H. Yagi," Proc. IEEE, vol. 85, no. 11, pp. 1857-1863, Nov. 1997.
[23] P. Y. Qin, Y. J. Guo, and C. Ding, "A beam switching quasi-Yagi dipole antenna," IEEE Trans. Antennas Propag., vol. 61, no. 10, pp. 4891-4899, Oct. 2013.

[24] P. Y. Qin, A. R. Weily, Y. J. Guo, T. S. Bird, and C. H. Liang, "Frequency Reconfigurable Quasi-Yagi Folded Dipole Antenna," IEEE Trans. Antennas Propag., vol. 61, no. 10, pp. 4891-4899, Oct. 2013. 